\title{
CALB1 enhances the interaction between p53 and MDM2, and inhibits the senescence of ovarian cancer cells
}

\author{
LONG-QIAO CAO $^{1}$, YA-NAN WANG ${ }^{2}$, MING LIANG $^{2}$ and MEI-ZHOU PAN ${ }^{3}$ \\ ${ }^{1}$ Reproductive Medical Center, The First People's Hospital of Jining, Jining, Shandong 272100; \\ ${ }^{2}$ Reproductive Medical Center, The Second Hospital Affiliated to Shandong University of Traditional Chinese Medicine; \\ ${ }^{3}$ Department of Urology Surgery, The Seventh People's Hospital of Jinan, Jinan, Shandong 251400, P.R. China
}

Received July 13, 2018; Accepted March 20, 2019

DOI: $10.3892 / \mathrm{mmr} .2019 .10212$

\begin{abstract}
Numerous studies have demonstrated the association between senescence and cancer. However, the molecular mechanism regulating senescence in ovarian cancer remains unknown. In the present study, the protein expression level of calbindin 1 (CALB1) in ovarian cancer was examined using western blot and immunohistochemistry. The function of CALB1 in ovarian cancer cells was examined using MTT assay, anchorage-independent growth assay and senescence assay. The molecular mechanisms underlying CALB1 function were investigated using immunoprecipitation and pull-down assays. In the present study, the expression of CALB1 was found to be increased in ovarian cancer. Overexpression of CALB1 promoted the proliferation and colony formation of ovarian cancer cells and inhibited senescence by modulating the expression levels of p21 and p27. Knockdown of CALB1 inhibited the proliferation and colony formation of ovarian cancer cells. Mechanistically, co-immunoprecipitation assays revealed that CALB1 interacts with MDM2 proto-oncogene (MDM2) and promoted the interaction between p53 and MDM2. Collectively, the present study suggested that CALB1 may act as an oncogene in ovarian cancer by inhibiting the p53 pathway.
\end{abstract}

\section{Introduction}

Ovarian cancer is one of the most common gynecological malignancies. Ovarian cancer can be resistance to chemotherapy, radiotherapy and targeted therapies $(1,2)$. Mutations of p53 and KRAS are common in ovarian cancer $(3,4)$. Understanding the molecular mechanism underlying ovarian cancer may facilitate the development of novel therapies.

Correspondence to: Professor Mei-Zhou Pan, Department of Urology Surgery, The Seventh People's Hospital of Jinan, 9 Xinyuan Avenue, Jinan, Shandong 251400, P.R. China

E-mail: panmeizhou2018@163.com

Key words: calbindin 1, p53, MDM2 proto-oncogene, senescence, ovarian cancer
Cellular senescence induces cell cycle arrest following cellular stress (5). A previous study observed that senescence is an important tumor-suppressive mechanism (6). Furthermore, accumulating evidence has demonstrated that p53, p21 [encoded by the cyclin dependent kinase inhibitor (CDKN)1A gene], p16 (encoded by CDKN2A) and retinoblastoma protein may have principal roles in regulating senescence (7). Genetic mutations in the p53 gene or downregulation of p53 caused by an increase in the expression level of the p53 ubiquitin ligase MDM2 proto-oncogene (MDM2) were identified as mechanisms that suppress senescence, and these processes were observed to cause therapeutic resistance (8).

A previous study observed that senescence occurs in ovarian cancer (9). However, whether senescence promotes the progression of ovarian cancer remains unclear $(10,11)$. A number of previous studies have demonstrated that chemotherapy drugs induce cellular senescence in tumor cells $(12,13)$. A recent study demonstrated that ovarian cancer cells promote hepatocyte growth factor-dependent senescence of peritoneal mesothelial cells, which may be involved in the formation of a metastatic niche for ovarian cancer cells within the peritoneal cavity $(14,15)$. Therefore, characterization of the mechanisms underlying senescence in ovarian cancer may facilitate the development of novel treatments.

Calbindin 1 (CALB1) is a member of the calcium-binding protein superfamily that includes calmodulins and troponin C (16). CALB1 contains four active calcium-binding domains and two modified domains that are unable to bind calcium (17). CALB1 was demonstrated to regulate calcium influx following the activation of glutamate receptors (18). Furthermore, genetic mutations in CALB1 gene have been observed in patients with Huntington disease (19). However, the role of CALB1 in cancer remains unknown.

In the present study, the expression pattern of CALB1 in ovarian cancer was examined. Additionally, the mechanisms associated with the role of CALB1 in the progression of this malignancy were investigated.

\section{Materials and methods}

Cell culture and transfection. Ovarian cancer cell lines (OVCA429, OVCA433 and OVCAR3) and normal ovarian epithelial cells (IOSE144) were purchased from The Cell 
Bank of Shanghai Institutes for Biological Science. Cells were maintained in DMEM (Gibco; Thermo Fisher Scientific, Inc.) supplemented with 10\% FBS (Gibco; Thermo Fisher Scientific, Inc.), $100 \mathrm{U} / \mathrm{ml}$ penicillin and $100 \mathrm{mg} / \mathrm{ml}$ streptomycin (Thermo Fisher Scientific, Inc.) in an incubator with 5\% $\mathrm{CO}_{2}$ at $37^{\circ} \mathrm{C}$.

In total, $10^{6}$ cells were plated in each dish $18 \mathrm{~h}$ before transfection. A total of $8 \mu \mathrm{g}$ plasmid was transfected into ovarian cancer cells using Lipofectamine 2000 (Invitrogen; Thermo Fisher Scientific, Inc.) according to the manufacture's protocol. Cells were incubated with antibiotics $(100 \mathrm{U} / \mathrm{ml}$ penicillin and $100 \mathrm{mg} / \mathrm{ml}$ streptomycin) for 3 days, and the resistant cells were pooled and used for the subsequent experiments.

Clinical samples. In total, 30 ovarian cancer samples and paired non-cancerous tissues were collected from patients who underwent surgery at The First People's Hospital of Jining (Jining, China) between April 2009 and March 2015. No treatment was performed prior to surgery. Written informed consent was obtained prior to the surgery. The collected tissues were stored in liquid nitrogen. The present study was approved by The Ethics Committee of The First People's Hospital of Jining.

Western blot analysis. The proteins were extracted from tissues and cell lines using RIPA lysis buffer (Cell Signaling Technology, Inc.), the protein concentration was measured by bicinchoninic acid assay. In total, $20 \mu \mathrm{g}$ protein was loaded in each lane. Proteins were separated by $8 \%$ SDS-PAGE (Sangon Biotech Co., Ltd.). Subsequently, the proteins were transferred onto a PVDF membrane (EMD Millipore). Following blocking with 5\% BSA (Sangon Biotech Co.,Ltd.) for $1 \mathrm{~h}$ at room temperature, the membranes were incubated with primary antibodies overnight at $4^{\circ} \mathrm{C}$. The membranes were subsequently washed with TBS-Tween-20 $(0.5 \%)$ and incubated with the appropriate horseradish peroxidase-conjugated secondary antibody (cat. no. 7074; Cell Signaling Technology, Inc.) for $1 \mathrm{~h}$ at room temperature. The proteins were visualized using an enhanced chemiluminescence kit (Pierce; Thermo Fisher Scientific, Inc.). Antibodies anti-CALB1 (1:3,000; cat. no. ab108404), p16 (1:3,000; cat. no. ab51243), p21 (1:3,000; cat. no. ab218311), p27 (also known as CDKN1B; 1:3,000; cat. no. ab32034), Tubulin (1:6,000; cat. no. ab210797), glutathione S-transferase (GST; 1:5,000; cat. no. ab111947), human influenza hemagglutinin (HA)-tag (1:5,000; cat. no. ab9110) and Flag (1:5,000; cat. no. ab49763) were purchased from Abcam. Anti-GAPDH $(1: 5,000$; cat. no. 5174$)$, myc $(1: 1,000$; cat. no. 2276$), \beta$-actin (1:5,000; cat. no. 3700$)$ and MDM2 (1:1,000; cat. no. 86934) were purchased from Cell Signaling Technology, Inc.

Immunohistochemistry (IHC). The 5- $\mu \mathrm{m}$-thick sections were deparaffinized and rehydrated using xylene $(100 \%)$ and descending ethanol series (75-100\%). Each incubation was performed for $10 \mathrm{~min}$ at room temperature. Subsequently, $0.3 \% \mathrm{H}_{2} \mathrm{O}_{2}$ for $10 \mathrm{~min}$ at room temperature was used to block endogenous peroxidase activity. Subsequently, the antigens were retrieved using sodium citrate $(\mathrm{pH} 6.0,0.01 \mathrm{M})$ at $98^{\circ} \mathrm{C}$ for $20 \mathrm{~min}$ ). Non-specific binding was blocked using $5 \%$ BSA (Sangon Biotech Co., Ltd.) at room temperature for $1 \mathrm{~h}$. The sections were stained with CALB1 primary antibody (Abcam) at $4^{\circ} \mathrm{C}$ overnight and visualized with the appropriate horseradish-conjugated secondary antibody using an EnVision system (Agilent Technologies, Inc.) according to the manufacturer's protocol. Subsequently, the slides were developed with 3,3'-diaminobenzidine at room temperature for $4 \mathrm{~min}$ and counterstained with hematoxylin at room temperature for $4 \mathrm{~min}$. The staining intensity and the protein expression levels were evaluated using the Vectra2 system (PerkinElmer, Inc.). The staining was assessed using the $\mathrm{H}$-score as previously described (20), which was calculating by multiplying the percentage of positive cells by the staining intensity of the tumor cells (ranging between 0 and 3 ).

Senescence-associated $\beta$-galactosidase ( $\beta$-gal) staining. Cells at $80 \%$ confluence were washed twice with PBS and fixed with $4 \%$ formaldehyde for $5 \mathrm{~min}$ at room temperature. Subsequently, cells were incubated at $37^{\circ} \mathrm{C}$ with $\beta$-gal staining solution (Beyotime Institute of Biotechnology) overnight according to the manufacturer's protocol. Stained cells were visualized using an inverted phase contrast light microscope (Olympus Corporation). In total, $>300$ random cells were analyzed per sample, and percentages of stained cells were calculated.

GST pull-down. The coding sequence of CALB1 was cloned into the expression vector pGEX-4T-1 (Clontech Laboratories, Inc.). A PCR was performed using cDNA derived from OVCA429 cells as template. The primers used to clone CALB1 were as follows: Forward, 5'-ATGGCAGAATCCCACCTG CA-3' and reverse, 5'-CTAGTTATCCCCAGCACAGAG-3'. The PCR was performed using KOD polymerase (Takara Bio, Inc.). The thermocycling conditions of the PCR were as follows: Initial denaturation at $95^{\circ} \mathrm{C}$ for $5 \mathrm{~min}$, followed by 35 cycles of $94^{\circ} \mathrm{C}$ for $30 \mathrm{sec}, 56^{\circ} \mathrm{C}$ for $1 \mathrm{~min}, 72^{\circ} \mathrm{C}$ for $2 \mathrm{~min}$, with a final extension at $72^{\circ} \mathrm{C}$ for $10 \mathrm{~min}$. The fusion protein GST-CALB1 was purified as previously described (21). The whole cell lysate of OVCA429 cells was extracted using a lysis buffer (containing $50 \mathrm{mM}$ Tris- $\mathrm{HCl} \mathrm{pH} \mathrm{7.5,150} \mathrm{mM} \mathrm{NaCl}, 0.1 \%$ Tergitol-type NP-40 and a protease inhibitor cocktail). The protein concentration was determined using the bicinchoninic acid assay. Subsequently, $5 \mu \mathrm{g}$ GST-CALB1 fusion protein and $500 \mu \mathrm{g}$ total protein from OVCA429 cells were incubated overnight at $4^{\circ} \mathrm{C}$. In total, $50 \mu \mathrm{l}$ Glutathione Sepharose 4B beads (GE Healthcare) were added to the samples and incubated at $4^{\circ} \mathrm{C}$ for $1 \mathrm{~h}$ to allow the binding between the beads and the GST fusion protein. Following three washes with lysis buffer, the proteins were eluted in Laemmli buffer and analyzed by SDS-PAGE as aforementioned.

Immunoprecipitation assay. Cells were lysed with RIPA buffer (Cell Signaling Technology, Inc.). Following centrifugation at $4^{\circ} \mathrm{C}$ for $20 \mathrm{~min}$ at $12,000 \mathrm{x} \mathrm{g}$, the supernatant of the cell lysate was incubated with a primary antibody overnight at $4^{\circ} \mathrm{C}$. Subsequently, the supernatant was incubated with $50 \mu \mathrm{l}$ protein $\mathrm{A}$ beads (GE Healthcare) for $4 \mathrm{~h}$ at $4^{\circ} \mathrm{C}$, and the beads were washed with RIPA buffer three times. The immunoprecipitated proteins were mixed with Laemmli (Cell Signaling Technology, Inc.) buffer and boiled for $5 \mathrm{~min}$ at $100^{\circ} \mathrm{C}$ prior to western blot analysis as aforementioned.

Plasmids. The coding sequence of CALB1 was amplified by PCR and inserted into the expression vector pcDNA3.1 
A

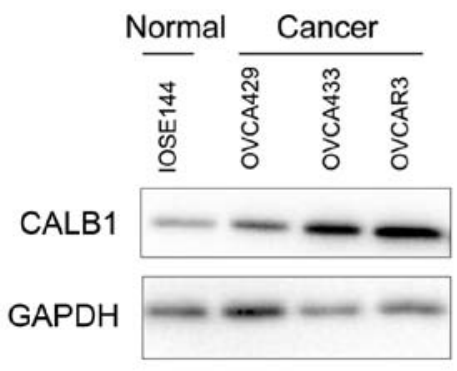

B

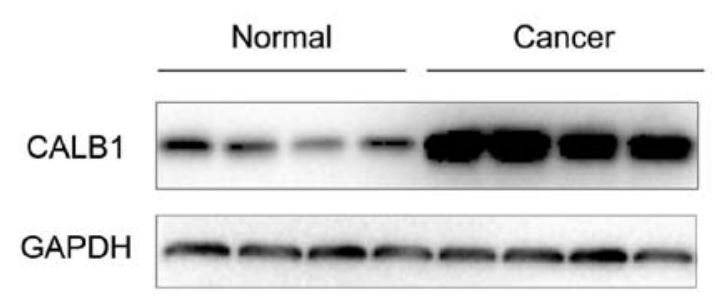

C
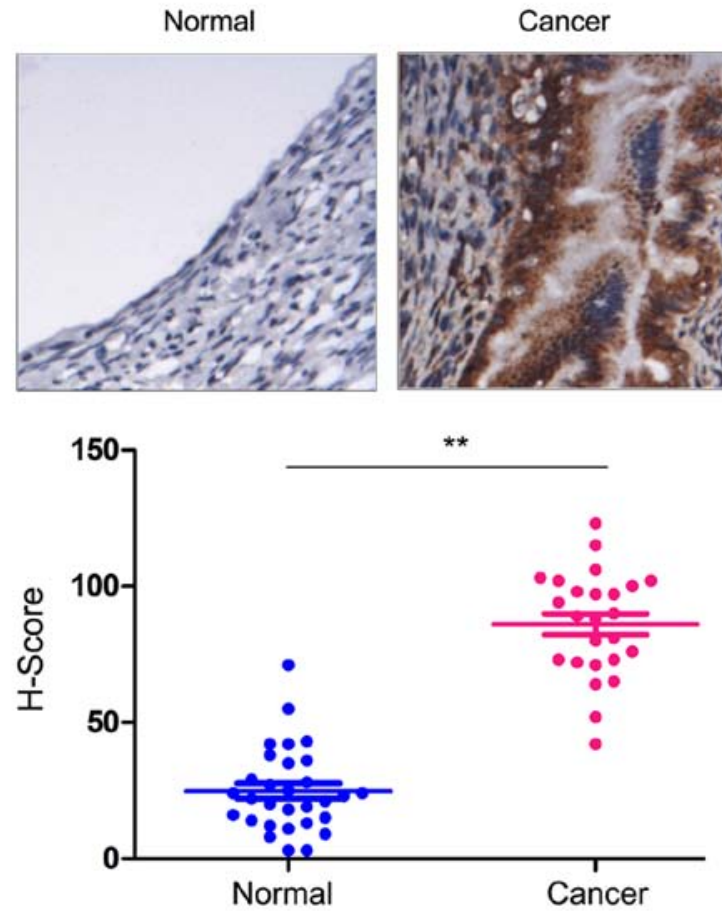

Cancer

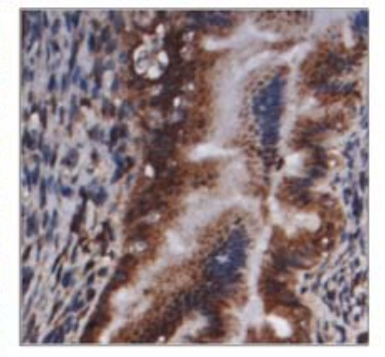

D

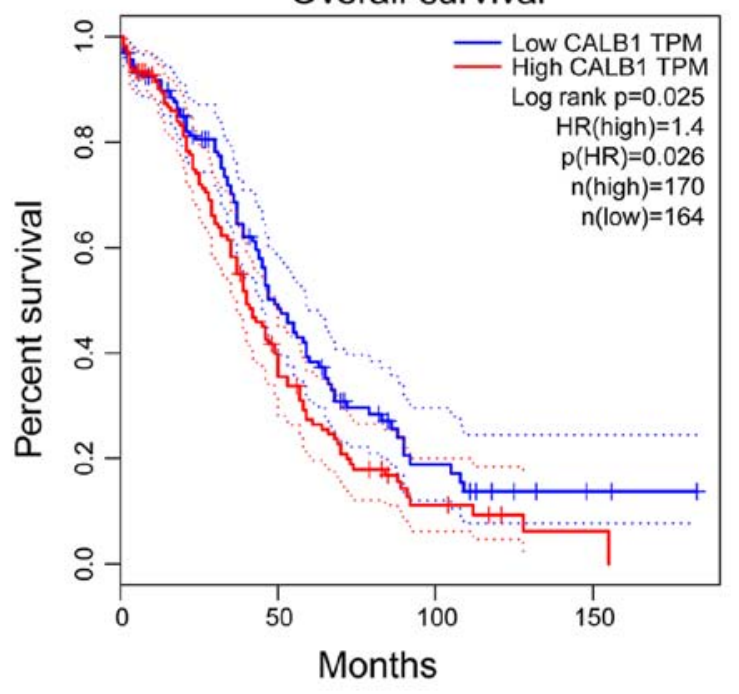

Figure 1. CALB1 is upregulated in ovarian cancer. (A) Western blot analysis was performed to examine the protein expression level of CALB1 in normal and ovarian cancer cells. (B) Western blot analysis was performed to examine the protein expression level of CALB1 in ovarian cancer tissues and adjacent non-cancerous tissues. (C) Immunohistochemical analysis was performed to examine the expression of CALB1 in 30 ovarian cancer and adjacent non-cancerous tissues. The staining of CALB1 was quantified. Magnification, x20. (D) The Gene Expression Profiling Interactive Analysis database was investigated to analyze the association between the expression level of CALB1 and survival rates of patients with ovarian cancer. ${ }^{* *} \mathrm{P}<0.01$. CALB1, calbindin 1; TPM, transcripts per million; H-score, histochemical score.

(Clontech Laboratories, Inc.) to construct the myc-tagged CALB1. The coding sequence of MDM2 was amplified by PCR and inserted into the expression vector pCMVTag2B (Clontech Laboratories, Inc.) to construct the Flag-tagged MDM2. The coding sequence of p53 was amplified by PCR and inserted into expression vector pCMV-HA (Clontech Laboratories, Inc.) to construct the HA-tagged p53. The cDNA was synthesized using RNA extracted from ovarian cancer cells as aforementioned. The primer sequences for MDM2 and p53 were as follows: MDM2 forward, 5'-ATGTGCAAT ACCTACATGTCTGTACC-3', MDM2 reverse, 5'-CTAGGG GAAATAAGTTAGCACAA-3'; P53 forward, 5'-ATGGAG GAGCCGCAGTCAGATCCTAGCG-3' and P53 reverse, 5'-TCAGTCTGAGTCAGGCCCTTCTGT-3'. The PCR was performed using KOD polymerase (Takara Bio, Inc.). The thermocycling conditions of the PCR were as follows: Initial denaturation at $95^{\circ} \mathrm{C}$ for $5 \mathrm{~min}$, followed by 35 cycles of $94^{\circ} \mathrm{C}$ for $30 \mathrm{sec}, 56^{\circ} \mathrm{C}$ for $1 \mathrm{~min}$ and $72^{\circ} \mathrm{C}$ for $2 \mathrm{~min}$, with a final extension at $72^{\circ} \mathrm{C}$ for $10 \mathrm{~min}$.
Knockdown of CALB1 expression. To knockdown CALB1, P21 and $\mathrm{P} 27$, lentiviral particles $\left(10^{8} \mathrm{PFU}\right)$ containing short hairpin (sh)-control or sh-CALB1, sh-P21 and sh-P27 (Shanghai GeneChem Co., Ltd.) were used to infect cells at $60 \%$ confluence for $24 \mathrm{~h}$, and cells exhibiting stable knockdown were selected using DMEM containing puromycin $(2 \mu \mathrm{g} / \mathrm{ml})$ for $\geq 1$ week. The sequences for sh-CALB1 were as follows: sh-CALB1 \#1, 5'-AATCCCACCTGCAGTCATCCC-3'; sh-CALB1 \#2, 5'-AATATGATACTGACCACAGTG-3'. The sequences for the additional sh-RNAs were as follows: sh-con, 5'-ACCACATCGCGTCTACACCTC-3'; sh-P21, 5'-AACCGG CTGGGGATGTCCGTC-3'; sh-P27, 5'-AAGAGTTAACCC GGGACTTGG-3'. In the senescence assay, only one sh-RNA for each gene was used. Only the sh-P21/P27 that was used in the transfection efficiency experiment was used in the senescence assay experiments.

MTT assay. Cells were plated in 96-well plates at a density of $1 \times 10^{3}$ cells/well. Cell proliferation was determined using the 

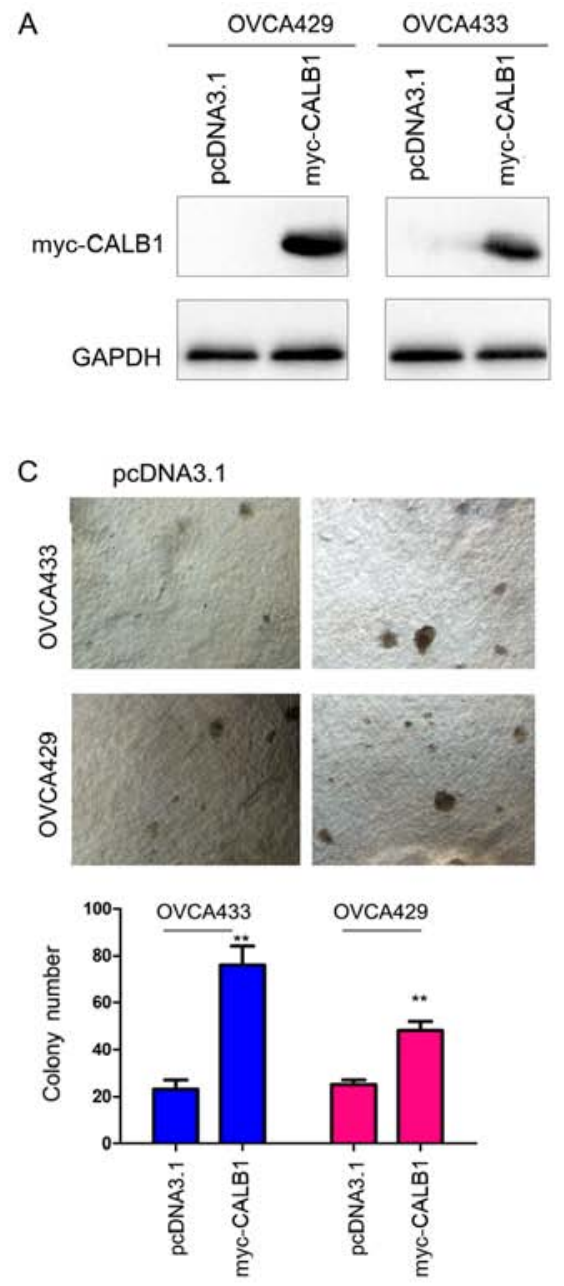

B
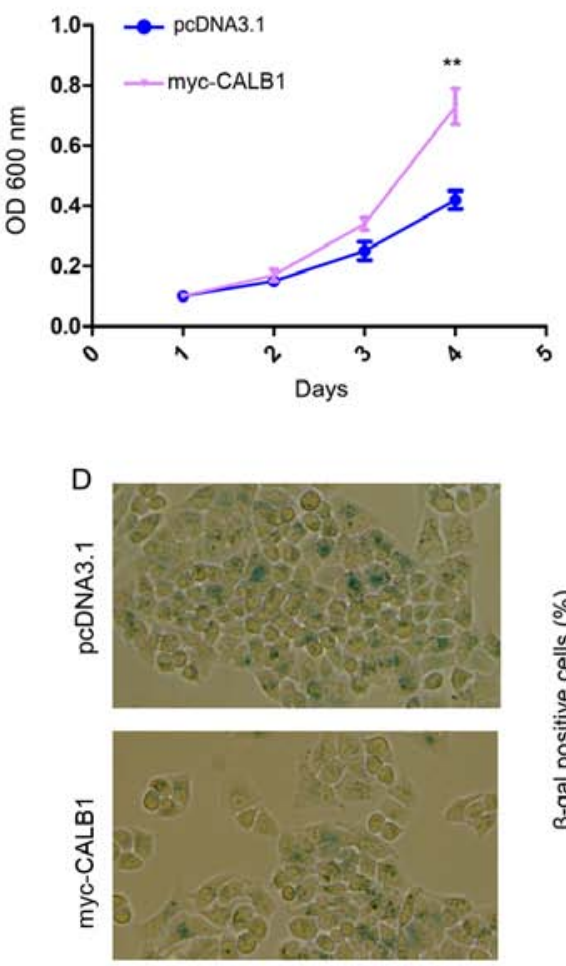

OVCA433

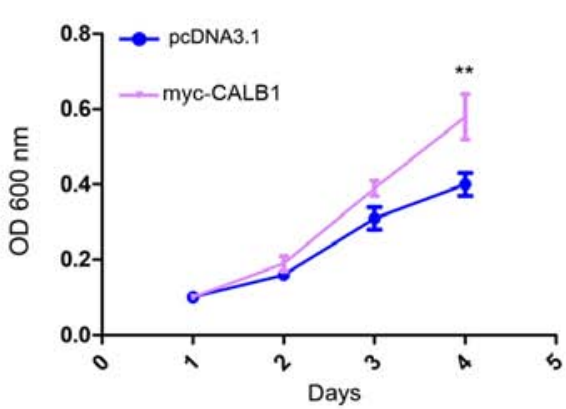

Figure 2. CALB1 promotes the proliferation and inhibits the senescence of ovarian cancer cells. (A) Western blot analysis was performed to examine the protein expression level of myc-tagged CALB1 in OVCA429 and OVCA433 cells. (B) MTT assay was performed to examine the effects of CALB1 overexpression on the proliferation of OVCA429 and OVCA433 cells. (C) Soft agar assay was performed to examine the effects of CALB1 overexpression on the anchorage-independent proliferation of OVCA429 and OVCA433 cells. (D) $\beta$-gal staining was performed on cells overexpressing CALB1. Magnification, 20. ${ }^{*} \mathrm{P}<0.05,{ }^{* *} \mathrm{P}<0.01$ vs. pcDNA3.1. CALB1, calbindin 1; OD, optical density; $\beta$-gal, $\beta$-galactosidase.

MTT colorimetric assay (R\&D Systems, Inc.) over 1 week. Every other day, cell proliferation was determined following incubation with the MTT solution (50 $\mu \mathrm{g} /$ well) for $4 \mathrm{~h}$. Following the MTT incubation, the purple formazan crystals were dissolved using DMSO (Sigma-Aldrich; Merck KGaA) and proliferation was analyzed at a wavelength of $540 \mathrm{~nm}$. All experiments were performed in triplicate.

Soft agar colony formation assay. For the soft agar assay, 12-well plates were coated with solidified DMEM-agarose [0.5\% agarose (Sangon Biotech Co., Ltd.) in DMEM supplemented with $10 \%$ FBS]. Subsequently, 2x $10^{3}$ cells/well suspended in a DMEM-agarose liquid solution $(0.35 \%$ agarose in DMEM supplemented with $10 \%$ FBS) were added in the pre-coated wells. Following 14 days of incubation, the colonies were counted and measured using a light microscope (magnification, 20x). All experiments were performed $\geq 3$ times.

Gene Expression Profiling Interactive Analysis tool (GEPIA) database analysis. GEPIA (http://gepia.cancer-pku.cn/) is a tool used to deliver fast and customizable functionalities based on TCGA and GTEx databases (22), including differential expression analysis, profiling plotting, correlation analysis, patient survival analysis and similar gene detection, as previously described (19). In the present study, GEPIA was used to investigate the association of CALB1 with disease-free survival rates of patients with ovarian cancer. Patients were grouped into high expression group and low expression group according to the median value of gene expression.

Statistical analysis. Data are presented as the mean \pm SEM. Each experiment was repeated three times. Two-tailed Student's t-test was performed to compare data between two groups. One-way ANOVA followed by Student-Newman-Keuls post hoc test was used to compare multiple groups. Kaplan-Meier analysis and log-rank tests were used for the survival analyses. Data were analyzed using the Prism software 5.0 (GraphPad Software, Inc.). $\mathrm{P}<0.05$ was considered to indicate a statistically significant difference.

\section{Results}

Expression level of CALBI is increased in ovarian cancer. To examine the expression of CALB1 in ovarian cancer, the 
A

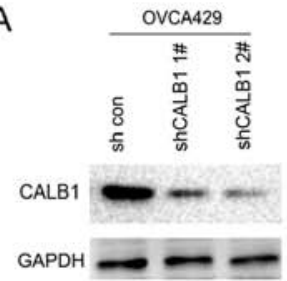

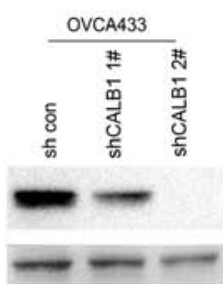

C
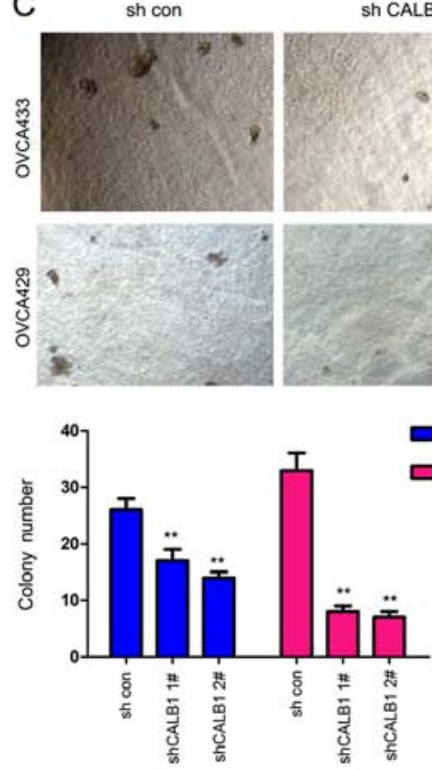

ovcaA33

OVCA429
B

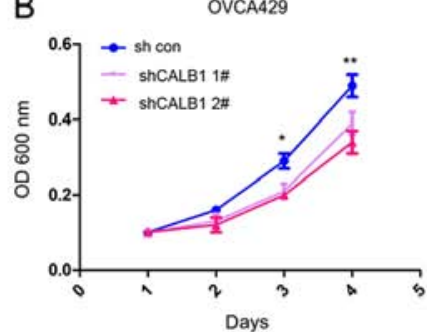

sh CALB 2\#
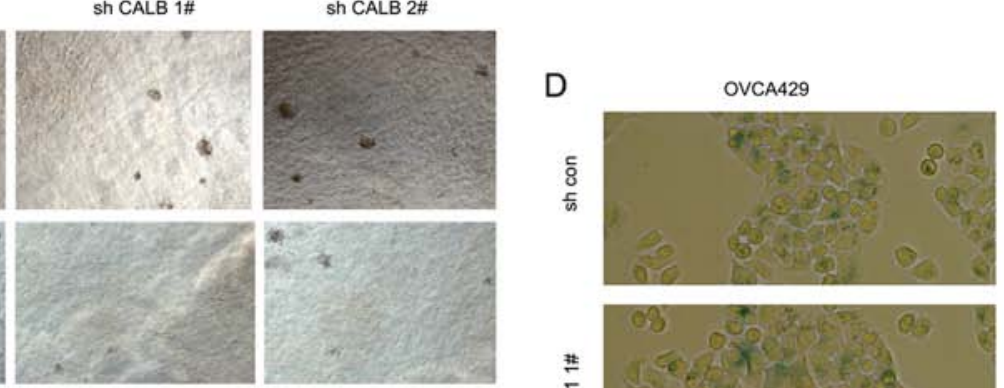
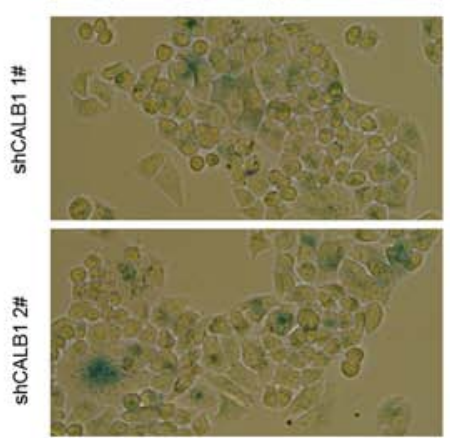
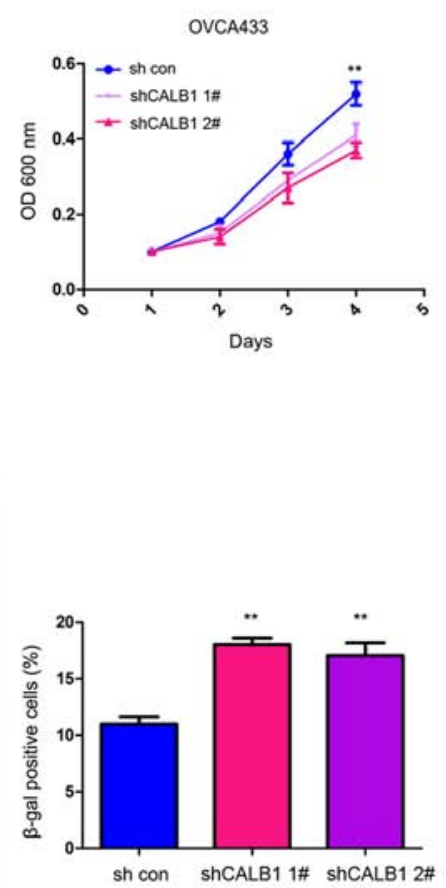

Figure 3. CALB1 knockdown promotes senescence of ovarian cancer cells. (A) Western blot analysis was performed to examine the expression of CALB1 in OVCA429 and OVCA433 cells following CALB1 knockdown. (B) MTT assay was performed to examine the effects of CALB1 knockdown on the proliferation of OVCA429 and OVCA433 cells. (C) Soft agar assay was performed to examine the effects of CALB1 knockdown on the anchorage-independent proliferation of OVCA429 and OVCA433 cells. (D) $\beta$-gal staining was performed following CALB1 knockdown. Magnification, $\mathrm{x} 20$. ${ }^{*} \mathrm{P}<0.05,{ }^{* * *} \mathrm{P}<0.01 \mathrm{vs}$. sh con. CALB1, calbindin 1 ; sh, short hairpin; con, negative control; OD, optical density; $\beta$-gal, $\beta$-galactosidase.

protein expression level of CALB1 was assessed in healthy and ovarian cancer cells. The present results demonstrated that the protein expression level of CALB1 was increased in cancer cells compared with the normal cell line (Fig. 1A). Subsequently, the protein expression level of CALB1 was analyzed in ovarian cancer samples and adjacent non-cancerous tissues using western blot analysis and IHC. The present results suggested that the protein expression level of CALB1 was increased in cancer tissues (Fig. 1B and C). Furthermore, the analysis of the Gene Expression Profiling Interactive Analysis database (gepia. cancer-pku.cn/detail.php?gene=CALB1) demonstrated that an increased expression of CALB1 was associated with a reduced survival rate (Fig. 1D). The present results suggested that the protein expression level of CALB1 is upregulated in ovarian cancer cell lines and tissues, and its expression level may be associated with poor prognosis in patients with ovarian cancer.

CALB1 promotes proliferation and inhibits senescence of ovarian cancer cells. To examine the biological functions of CALB1 in ovarian cancer, CALB1 was overexpressed in OVCA429 and OVCA433 cells (Fig. 2A). MTT and soft agar assays were used to examine the effects of CALB1 overexpression on the proliferation of ovarian cancer cells. Overexpression of CALB1 increased the proliferation of cancer cells in liquid medium after 4 days and the anchorage-independent cell proliferation in soft agar, as assessed by MTT and colony formation assay, respectively (Fig. 2B and C). Notably, the $\beta$-gal staining results suggested that the number of control cells positive for $\beta$-gal was higher than cells overexpressing CALB1 (Fig. 2D), suggesting that CALB1 may inhibit senescence in ovarian cancer cells.

To further investigate the biological functions of CALB1 in ovarian cancer cells, the expression of CALB1 was silenced using two sh-RNA sequences targeting CALB1 in OVCA429 and OVCA433 cells (Fig. 3A). Knockdown of CALB1 inhibited the proliferation of ovarian cancer cells in liquid culture after 3 days in OVCA429 cells and after 4 days in both cell types (Fig. 3B). Additionally, knockdown of CALB1 decreased the colony formation of ovarian cancer cells in soft agar (Fig. 3C). Furthermore, following transfection with sh-CALB1, the number of senescent cells increased, as assessed by $\beta$-gal staining (Fig. 3D). Collectively, the present data suggested that CALB1 promoted proliferation and inhibited senescence of ovarian cancer cells.

p21 and p27 are involved in the sh-CALB1-induced senescence. The protein expression levels of various 
A

OVCA429
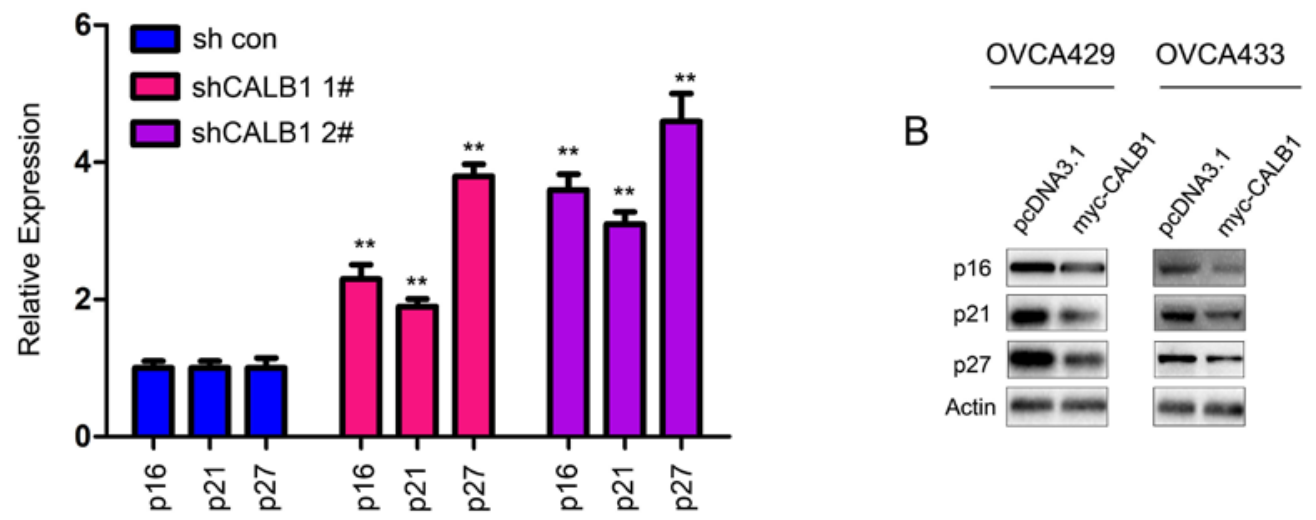

C
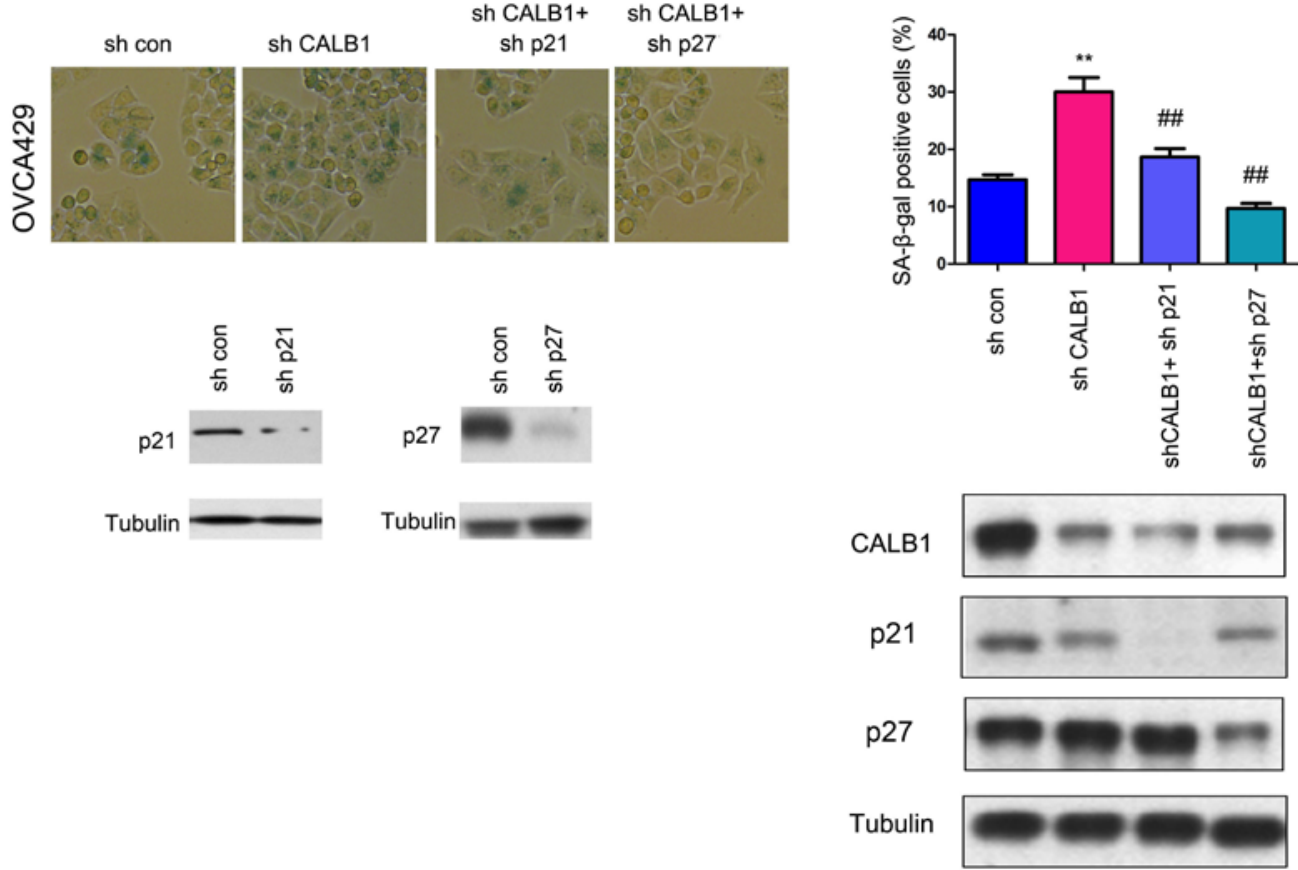

Figure 4. CALB1 knockdown promotes senescence via p21 and p27. (A) Quantitative PCR was performed to examine the mRNA expression levels of p16, p21 and p27. (B) Western blot analysis was performed to examine the protein expression levels of p16, p21 and p27. (C) Knockdown of p21 and p27 decreased cell senescence and the protein expression level of $\mathrm{p} 21$ and $\mathrm{p} 27$. Magnification, $\mathrm{x} 20 .{ }^{* *} \mathrm{P}<0.01$ vs. sh con; ${ }^{\# \#} \mathrm{P}<0.01$ vs. sh $\mathrm{CALB} 1$. sh, short hairpin; con, control; CALB1, calbindin 1; p16, cyclin-dependent kinase inhibitor 2A; p21, cyclin-dependent kinase inhibitor 1A; p27, cyclin-dependent kinase inhibitor 1B.

regulators of senescence were examined. In particular, the protein expression levels of p16, p21 and p27 were assessed by western blot analysis. Knocking down the expression of CALB1 significantly upregulated the mRNA expression levels of p16, p21 and p27 (Fig. 4A). Conversely, overexpression of CALB1 decreased the protein expression level of p16, p21 and p27 (Fig. 4B). Moreover, knocking down the expression of p21 and p27 abolished the senescence induced by sh-CALB1, as assessed by $\beta$-gal staining (Fig. 4C). The present data suggested that p21 and p27 were responsible for the increase in senescence induced by CALB1 knockdown.

CALB1 interacts with MDM2, and promotes the interaction between MDM2 and p53. Numerous previous studies have demonstrated that $\mathrm{p} 21$ and $\mathrm{p} 27$ are downstream target genes of p53 (23). Therefore, the interaction between CALB1 and other members of the $\mathrm{p} 53$ signaling pathway was investigated in the present study. In the GST pull down assay, the fusion protein GST-CALB1 interacted with endogenously expressed MDM2 (Fig. 5A), as confirmed by the co-immunoprecipitation assay (Fig. 5B and C). Furthermore, CALB1 enhanced the interaction between p53 and MDM2 (Fig. 5D), suggesting that CALB1 may promote the degradation of p53 via MDM2.

\section{Discussion}

Numerous previous studies have demonstrated that cellular senescence contributes to tumor suppression (24). However, 

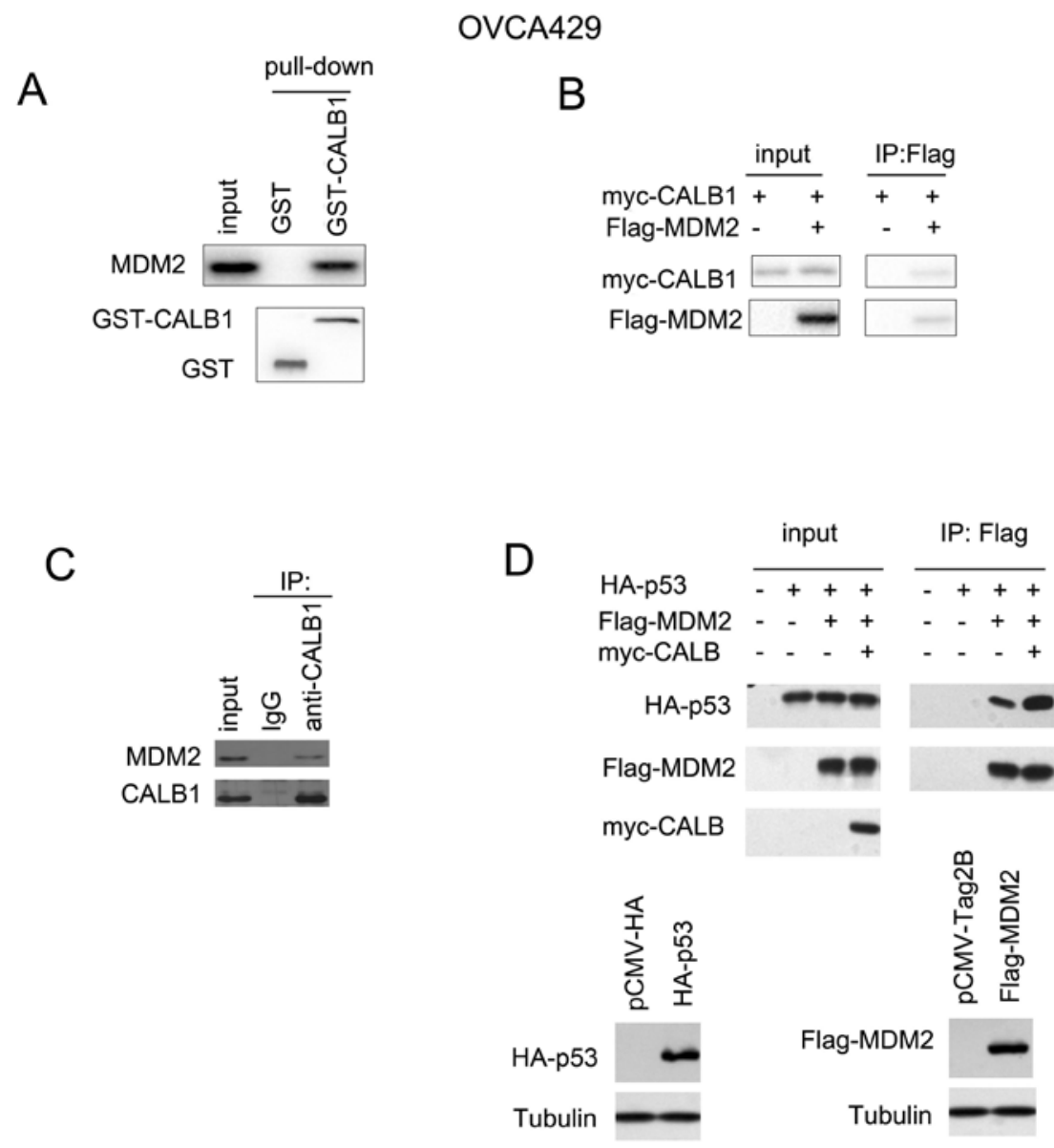

Figure 5. CALB1 interacts with MDM2. (A) GST pull down assay was performed using GST-CALB1 fusion protein. (B) Immunoprecipitation following overexpression of myc-CALB1 and Flag-MDM2 was performed to examine the interaction between CALB1 and MDM2. (C) Endogenous immunoprecipitation was performed to examine the interaction between CALB1 and MDM2. (D) Immunoprecipitation following overexpression of HA-p53, Flag-MDM2 and/or myc-CALB1 was performed to examine the effects of CALB1 overexpression on the interaction between p53 and MDM2. MDM2, MDM2 proto-oncogene; GST, glutathione S-transferase; CALB1, calbindin 1; IP, immunoprecipitation; HA, human influenza hemagglutinin.

the molecular mechanism underlying this process remains unclear. In the present study, CALB1 was identified to be upregulated in ovarian cancer. Functional analysis suggested that CALB1 promoted the proliferation and colony formation of ovarian cancer cells by inhibiting senescence. The molecular experiments demonstrated the interaction between MDM2 and CALB1, and CALB1 enhanced the interaction between p53 and MDM2, thus potentially leading to the degradation of the p53 tumor suppressor. In line with this hypothesis, CALB1 knockdown upregulated the expression levels of p16, p21 and p27, and overexpression of CALB1 decreased the protein expression level of p16, p21 and p27. The present results suggested that CALB1 may act as an oncogene in ovarian cancer.

The present study identified the oncogenic roles of CALB1 in ovarian cancer. To the best of our knowledge, a few previous studies have investigated the roles of CALB1 in carcinogenesis (25). The present study suggested that CALB1 may act as an oncogene in ovarian cancer, and CALB1 may represent a novel therapeutic target to treat ovarian cancer.

In the present study, knockdown of p21 and p27, two target genes of p53 (26), suppressed the senescence induced by CALB1 knockdown, which further emphasized the roles of p53 and its target genes in the process of senescence. It is established that ovarian cancer develops due to accumulation of multiple genetic mutations (27). In addition to KRAS, other regulators of senescence, such as p53 and p16, are frequently mutated in human ovarian cancer (28).

In summary, the present study suggested that CALB1 may act as an oncogene in ovarian cancer by inhibiting senescence, and suggested that CALB1 may be a novel therapeutic target for the treatment of ovarian cancer. Further experiments using a large number of clinical samples are required to investigate the potential to use CALB1 as an ovarian cancer biomarker.

\section{Acknowledgements}

Not applicable.

\section{Funding}

No funding was received.

\section{Availability of data and materials}

The datasets used and/or analyzed during the current study are available from the corresponding author on reasonable request. 


\section{Authors' contributions}

MP and ML designed the present study, and interpreted and analyzed the data. YW and LC performed the experiments. All authors read and approved the final manuscript.

\section{Ethics approval and consent to participate}

This study was approved by The Ethics Committee of The Seventh People's Hospital of Jinan, and written informed consent was obtained from all patients.

\section{Patient consent for publication}

All patients within this study provided consent for the publication of their data.

\section{Competing interests}

The authors declare that they have no competing interests.

\section{References}

1. Torre LA, Trabert B, DeSantis CE, Miller KD, Samimi G, Runowicz CD, Gaudet MM, Jemal A and Siegel RL: Ovarian cancer statistics, 2018. CA Cancer J Clin 68: 284-296, 2018.

2. Siegel RL, Miller KD and Jemal A: Cancer statistics, 2018. CA Cancer J Clin 68: 7-30, 2018.

3. Mori R, Futamura M, Morimitsu K, Saigo C, Miyazaki T and Yoshida K: The diagnosis of a metastatic breast tumor from ovarian cancer by the succession of a p53 mutation: A case report. World J Surg Oncol 15: 117, 2017.

4. Nakayama N, Nakayama K, Yeasmin S, Ishibashi M, Katagiri A, Iida K, Fukumoto M and Miyazaki K: KRAS or BRAF mutation status is a useful predictor of sensitivity to MEK inhibition in ovarian cancer. Br J Cancer 99: 2020-2028, 2008.

5. Bennett MR and Clarke MC: Basic research: Killing the old: Cell senescence in atherosclerosis. Nat Rev Cardiol 14: 8-9, 2016.

6. Burgess DJ: Senescence: Double or quit? Nat Rev Cancer 11: 389, 2011.

7. Mathon NF and Lloyd AC: Cell senescence and cancer. Nat Rev Cancer 1: 203-213, 2001.

8. Burgess DJ: Senescence: Tumorigenesis under surveillance. Nat Rev Cancer 12: 6, 2011.

9. Visser JA, Schipper I, Laven JS and Themmen AP: Anti-Mullerian hormone: An ovarian reserve marker in primary ovarian insufficiency. Nat Rev Endocrinol 8: 331-341, 2012.

10. Nardella C, Clohessy JG, Alimonti A and Pandolfi PP Pro-senescence therapy for cancer treatment. Nat Rev Cancer 11: 503-511, 2011.

11. Campisi J and d'Adda di Fagagna F: Cellular senescence: When bad things happen to good cells. Nat Rev Mol Cell Biol 8: 729-740, 2007
12. Killock D: Chemotherapy: Life gained, years lost? Nat Rev Clin Oncol 11: 303, 2014.

13. Collado M and Serrano M: Senescence in tumours: Evidence from mice and humans. Nat Rev Cancer 10: 51-57, 2010.

14. Li J, Mao Q, He J, She H, Zhang Z and Yin C: Human umbilical cord mesenchymal stem cells improve the reserve function of perimenopausal ovary via a paracrine mechanism. Stem Cell Res Ther 8: 55, 2017.

15. Mikuła-Pietrasik J, Uruski P, Pakuła M, Maksin K, Szubert S, Woźniak A, Naumowicz E, Szpurek D, Tykarski A and Książek K: Oxidative stress contributes to hepatocyte growth factor-dependent pro-senescence activity of ovarian cancer cells. Free Radic Biol Med 110: 270-279, 2017.

16. Jung EM, Choi KC and Jeung EB: Expression of calbindin-D28k is inversely correlated with proapototic gene expression in hydrogen peroxide-induced cell death in endometrial cancer cells. Int J Oncol 38: 1059-1066, 2011.

17. Goffigan-Holmes J, Sanabria D, Diaz J, Flock D and Chavez-Valdez R: Calbindin-1 expression in the hippocampus following neonatal hypoxia-ischemia and therapeutic hypothermia and deficits in spatial memory. Dev Neurosci: March 12, 2019 (Epub ahead of print).

18. Parkash J, Chaudhry MA and Rhoten WB: Calbindin-D28k and calcium sensing receptor cooperate in MCF-7 human breast cancer cells. Int J Oncol 24: 1111-1119, 2004.

19. Massouh M, Wallman MJ, Pourcher E and Parent A: The fate of the large striatal interneurons expressing calretinin in Huntington's disease. Neurosci Res 62: 216-224, 2008.

20. Cai Z, Qian ZY, Jiang H, Ma N, Li Z, Liu LY, Ren XX, Shang YR, Wang JJ, Li JJ, et al: hPCL3s promotes hepatocellular carcinoma metastasis by activating $\beta$-catenin signaling. Cancer Res 78: 2536-2549, 2018

21. Deng YZ, Yao F, Li JJ, Mao ZF, Hu PT, Long LY, Li G, Ji XD, Shi S, Guan DX, et al: RACK1 suppresses gastric tumorigenesis by stabilizing the $\beta$-catenin destruction complex. Gastroenterology 142: 812-823.e15, 2012.

22. Tang Z, Li C, Kang B, Gao G, Li C and Zhang Z: GEPIA: A web server for cancer and normal gene expression profiling and interactive analyses. Nucleic Acids Res 45: W98-W102, 2017.

23. Levine AJ: The p53 tumor suppressor gene and gene product. Princess Takamatsu Symp 20: 221-230, 1989.

24. Ohtani N, Mann DJ and Hara E: Cellular senescence: Its role in tumor suppression and aging. Cancer Sci 100: 792-797, 2009.

25. Jin C, Lin T and Shan L: Downregulation of Calbindin 1 by miR-454-3p suppresses cell proliferation in nonsmall cell lung cancer in vitro. Cancer Biother Radiopharm 34: 119-127, 2019.

26. Yang F, von Knethen A and Brune B: Modulation of nitric oxide-evoked apoptosis by the p53-downstream target p21(WAF1/CIP1). J Leukoc Biol 68: 916-922, 2000.

27. Labidi-Galy SI, Olivier T, Rodrigues M, Ferraioli D, Derbel O, Bodmer A, Petignat P, Rak B, Chopin N, Tredan O, et al: Location of mutation in BRCA2 gene and survival in patients with ovarian cancer. Clin Cancer Res 24: 326-333, 2018.

28. Schuyer M, van Staveren IL, Klijn JG, vd Burg ME, Stoter G, Henzen-Logmans SC, Foekens JA and Berns EM: Sporadic CDKN2 (MTS1/p16ink4) gene alterations in human ovarian tumours. Br J Cancer 74: 1069-1073, 1996. 